Expertenstandard wird aktualisiert "Dekubitusprophylaxe
in der Pflege"

- Der Expertenstandard Dekubitusprophylaxe steht zur zweiten Aktualisierung an. Für die Aktualisierung wird eine neue Literaturstudie zum aktuellen Wissens- und Erkenntnisstand in Auftrag gegeben und eine neue Expertenarbeitsgruppe einberufen. Der Aktualisierungszeitraum wird sich über ca. 18 Monate erstrecken. Im Rahmen der Aktualisierung sollen zudem Indikatoren für das interne Qualitätsmanagement entwickelt werden.

Für die fachliche Leitung der Expertenarbeitsgruppe wird eine/ein auf diesem Gebiet ausgewiesene/r Wissenschaftlerin/ Wissenschaftler mit Expertise zum Thema und der Leitung von Projektgruppen gesucht. Die wissenschaftliche Leitung der Expertenarbeitsgruppe ist verantwortlich für das wissenschaftliche Niveau des Expertenstandards, der Kommentierungen und der Literaturanalyse unter Berücksichtigung der Praxisbedingungen. Im Zusammenhang mit der Aktualisierung sollen auch Indikatoren auf Basis des aktualisierten Expertenstandards entwickelt werden, weshalb Erfahrungen in diesem Bereich von Vorteil sind. Wünschenswert ist die Zusicherung der Verfügbarkeit von zwei wissenschaftlichen Mitarbeitern/Mitarbeiterinnen mit einer entsprechenden Qualifikation für die Durchführung der Literaturrecherche nach anerkannten Verfahren. Für die Erstellung der Literaturstudie und die Personalkosten werden finanzielle Ressourcen zur Verfügung gestellt. Die wissenschaftliche Leitung der Expertenarbeitsgruppe erfolgt ehrenamtlich, lediglich Reisekosten im Zusammenhang mit der Aktualisierung des Expertenstandards werden erstattet.

Neben der fachlichen Expertise werden die Bewerberin/der Bewerber gebeten, eigene Interessen, Verbindungen zur Industrie oder Interessensverbänden offen zu legen.

Bewerbungen werden bis zum 05. Oktober 2015 an die folgende Anschrift erbeten: Deutsches Netzwerk für Qualitätsentwicklung in der Pflege (DNQP) an der Hochschule Osnabrück, Prof. Dr. Andreas Büscher, Postfach 19 40, 49009 Osnabrück oder dnqp@hs-osnabrueck.de
Pflegestärkungsgesetz II

\section{Demenzkranke erhalten Leistungen aus der Pflegeversicherung}

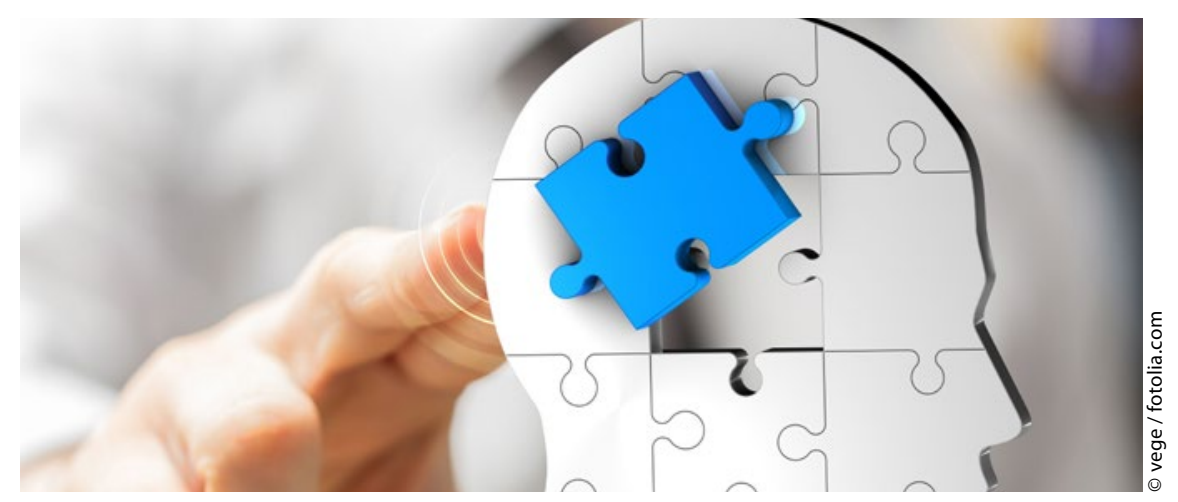

Das Bundeskabinett hat am 12. August die zweite Stufe der Pflegereform auf den Weg gebracht. Das Pflegestärkungsgesetz soll das Leistungsangebot für Pflegebedürftige und Pflegende verbessern. Zentraler Punkt ist ein neuer Pflegebedürftigkeitsbegriff, der Demenzkranken Anspruch auf die Leistungen einräumt wie Menschen mit körperlichen Beeinträchtigungen. Die bisherigen drei Pflegestufen werden auf fünf Pflegegrade ausgeweitet. Anfang des Jahres trat das erste Pflegestärkungsgesetz in Kraft. Mit beiden Reformen ist eine Erhöhung des Beitrags zur Pflegeversicherung verbunden - von 2,05\% auf 2,55 von 2017 an. Beide Erhöhungen bringen zusammen rund fünf Milliarden Euro für zusätzliche Leistungen. Bundesgesundheitsminister Gröhe (CDU) hatte seinen Entwurf zuletzt nochmals nachgebessert, unter anderem zugunsten pflegender Angehöriger. Der Deutsche Berufsverband für Pflegeberufe (DBfK) begrüßt die grundlegende Reform der Pflegeversicherung. „Der neue Pflegebedürftigkeitsbegriff ist pflegewissenschaftlich begründet und mit seiner Umsetzung gibt es mehr Gerechtigkeit bei der Begutachtung, auch für Menschen mit kognitiven Beeinträchtigungen", sagt DBfK-Bundesgeschäftsführer Franz Wagner. Die Pflegereform könne aber nur greifen, wenn sichergestellt wird, dass ausreichend Pflegende vorhanden sind. Perspektivisch bei den Angehörigen, aber schon ganz akut bei den Pflegefachpersonen gäbe es ein großes Kapazitätsproblem.

www.dbfk.de 